\title{
Neoplasia mucinosa papilar de la vía biliar: reporte de caso
}

\section{A Case Report of Biliary Tract Intraductal Papillary Mucinous Neoplasm}

Martín A. Gómez Z., MD, ${ }^{1}$ Javier Riveros V., MD, ${ }^{2}$ William Otero R., MD. ${ }^{3}$

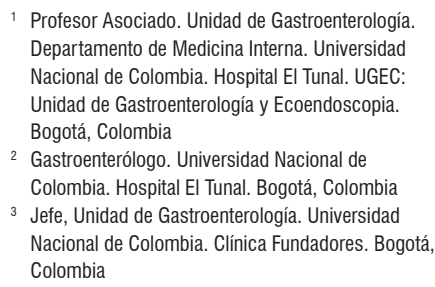

Profesor Asociado. Unidad de Gastroenterología. Departamento de Medicina Interna. Universidad Nacional de Colombia. Hospital El Tunal. UGEC: Unidad de Gastroenterología y Ecoendoscopia. Bogotá, Colombia

2 Gastroenterólogo. Universidad Nacional de Colombia. Hospital El Tunal. Bogotá, Colombia

3 Jefe, Unidad de Gastroenterología. Universidad Nacional de Colombia. Clínica Fundadores. Bogotá, Colombia

Fecha recibido: $10-11-14$

Fecha aceptado: 21-07-15

\begin{abstract}
Resumen
La neoplasia mucinosa papilar es una enfermedad muy rara que usualmente se describe como ubicada en la vía pancreática. Presentamos un caso de una mujer con esta enfermedad aunque localizada en la vía biliar, lo cual es aún más infrecuente y constituye el primer caso descrito en Colombia. Hacemos una revisión de la literatura y una descripción de los exámenes que se usaron para llegar al diagnóstico, que incluyeron la utilidad de una nueva herramienta como es el Spyglass.
\end{abstract}

\section{Palabras clave \\ Colestasis, neoplasia mucinosa, colangioscopia.}

\begin{abstract}
Papillary mucinous neoplasms are rare and are usually located in the pancreatic pathway. We present a case of a woman with this disease located in the bile duct, an even rarer occurrence. This is the first case in Colombia. We review the literature, describe the tests used for diagnosis, and include an evaluation of the usefulness of a new tool, the Spyglass.
\end{abstract}

Key words

Cholestasis, mucinous neoplasm, cholangioscopy.
La neoplasia mucinosa papilar de la vía biliar (NMPVB) es una patología muy rara (este es el primer caso reportado en Colombia y uno de los pocos casos encontrados en el mundo). Se asocia con sobreproducción de moco y mucobilia, y se incluyó dentro del espectro de las neoplasias biliares con hepatolitiasis. Inicialmente fue descrita como una variante de la neoplasia mucinosa papilar del páncreas (NMPP) por su gran similitud histológica (1): la NMPVB tiende a darse predominantemente en mujeres como nuestra paciente, clínicamente presenta un patrón colestásico por hiperproducción de moco en la vía biliar y una elevación en marcadores tumorales como el antígeno carcinoembrionario (ACE) y el antígeno carbohidrato (CA) 19-9 (2); por otra parte, la NMPP es más frecuente en hombres, solo presentan hipersecreción de mucina en el $66 \%$ de los casos, no se asocia con colestasis ni con elevación de bilirrubinas, y usualmente los marcadores tumorales son normales (3).

\section{PRESENTACIÓN DE CASO}

Paciente femenina de 58 años de edad, colecistectomizada, que consulta por cuadro compatible con coledocolitiasis. Se llevó a colangiopancreatografía retrógrada endoscópica (CPRE) extra institucional, la cual reportó extracción de cálculos, pero la paciente persistió con síntomas obstructivos y pérdida de peso, por esto se le realizaron marcadores tumorales (ACE y CA19-9 elevados), tomografía axial 
computarizada (TAC) y espectroscopia de resonancia magnética nuclear (C-RNM) sin aclarar la causa del cuadro, por lo que es remitida a nuestra institución. Dada la marcada obstrucción biliar, se decidió realizar una ecoendoscopia que señaló imágenes isoecoicas en la vía biliar, que no dejaban sombra acústica, compatibles con moco (figura 1), así que se lleva nuevamente a CPRE encontrando dilatación del colédoco (figura 2) y abundante moco sin cálculos que obstruyeran la vía biliar (figura 3). Como el diagnóstico aún no era claro, se llevó a coledocoscopia con Spyglass encontrando abundante moco (figura 4) en la vía biliar e irregularidad segmentaria de la pared del colédoco por múltiples papilas, por lo cual se tomó biopsia dirigida que confirmó el diagnóstico de NMPVB y se programó para quimioterapia, dada la multifocalidad de la lesión, con excelente evolución.

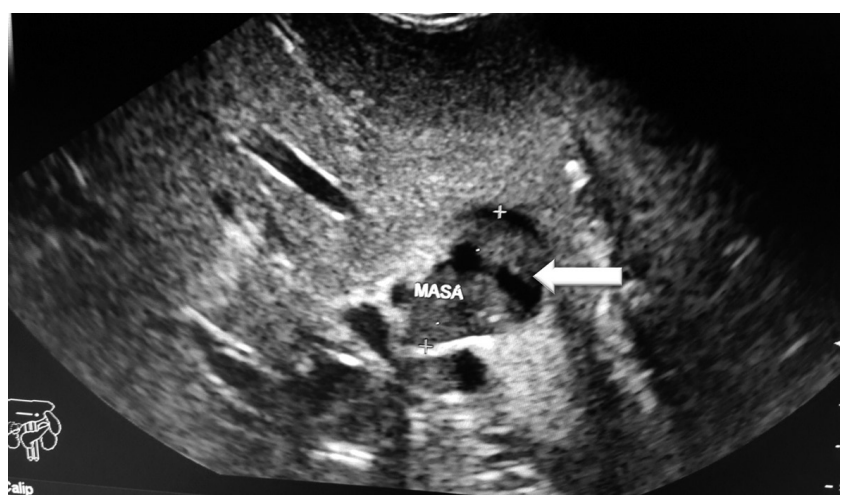

Figura 1. La flecha muestra una imagen isoecoica dentro de la vía biliar intrahepática que no deja sombra acústica, lo cual es sugestivo de una masa. Como se desplaza con los movimientos, es sospechosa de corresponder a moco dentro de la vía biliar, lo cual se confirmó posteriormente.

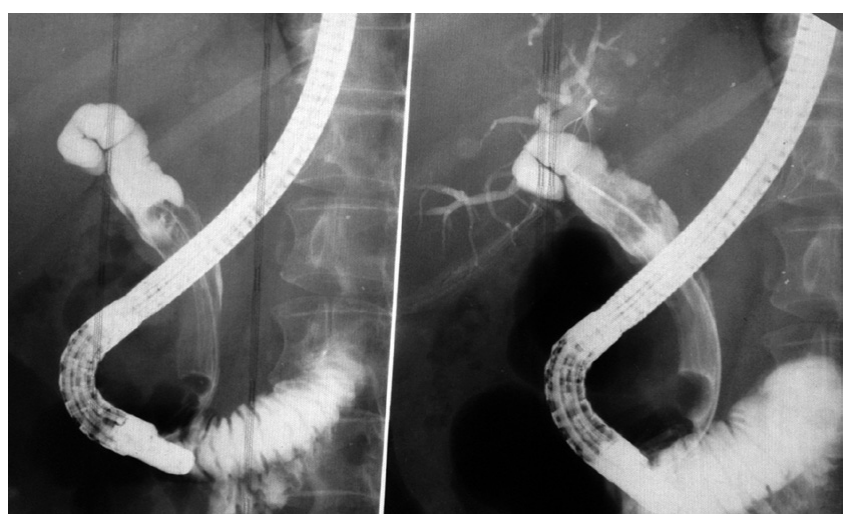

Figura 2. Se realiza CPRE con balón, y muestra que la vía biliar está marcadamente dilatada con imagen radiolúcida que ocupa todo el colédoco. Al retirar el balón se observa abundante moco.

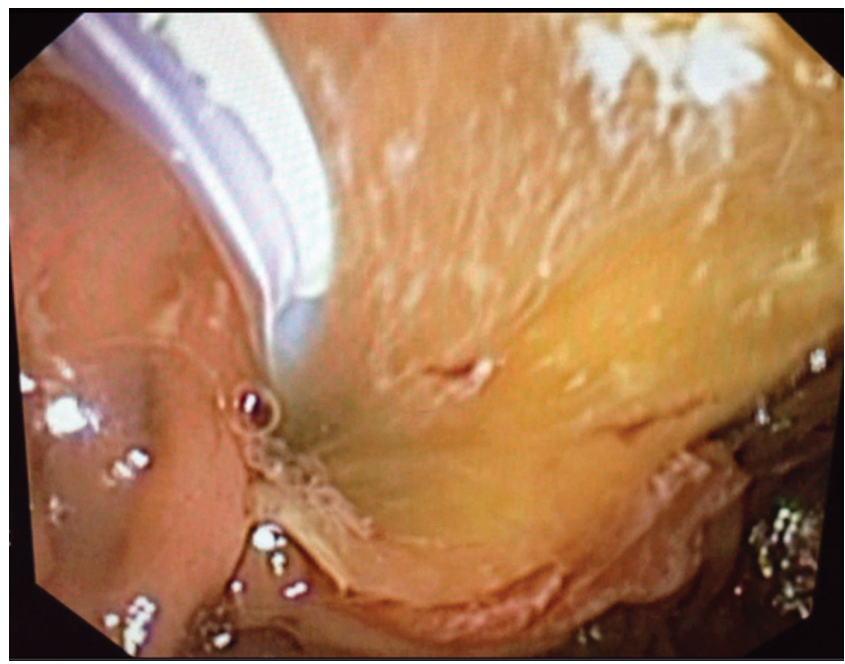

Figura 3. Se observa el balón en la parte superior, y en la inferior se ve abundante moco que es retirado con el balón.

\section{DISCUSIÓN}

Este trabajo presenta una causa rara de colestasis que debe tenerse en cuenta en el diagnóstico diferencial de la obstrucción biliar diferente a la litiasis, la cual es la causa más frecuente. Además muestra cómo técnicas modernas, como la ecoendoscopia, permitieron sospechar la causa de la obstrucción al sugerir la presencia de moco, y el Spyglass permitió confirmar la enfermedad al lograr una visualización directa de la pared del colédoco y observar las papilas productoras de moco, lo que permitió la toma dirigida de la biopsia.

La NMPVB se define como un tumor epitelial biliar que se caracteriza por la formación de papilas dentro del ducto biliar y la producción de moco, y se puede desarrollar en cualquier lugar a lo largo del árbol biliar, incluyendo los conductos biliares intra y extrahepáticos $(4,5)$. La hipersecreción de mucina lleva a la obstrucción y consecuentemente, a la dilatación de la vía biliar. Microscópicamente, la NMPVB se compone de papilas con vasos finos y células epiteliales neoplásicas que muestran un espectro de atipia citoarquitectónica que va desde la displasia de bajo grado hasta el carcinoma invasivo $(6,7)$; debido a estas características, la NMPVB se considera como una lesión premaligna que puede llevar a un colangiocarcinoma invasivo (8). En la clasificación de la Organización Mundial de la Salud (OMS) (9), la NMPVB se clasifica en: NMP intraepitelial de grado bajo o intermedio, NMP con alto grado de neoplasia intraepitelial, y NMP con un carcinoma invasivo 

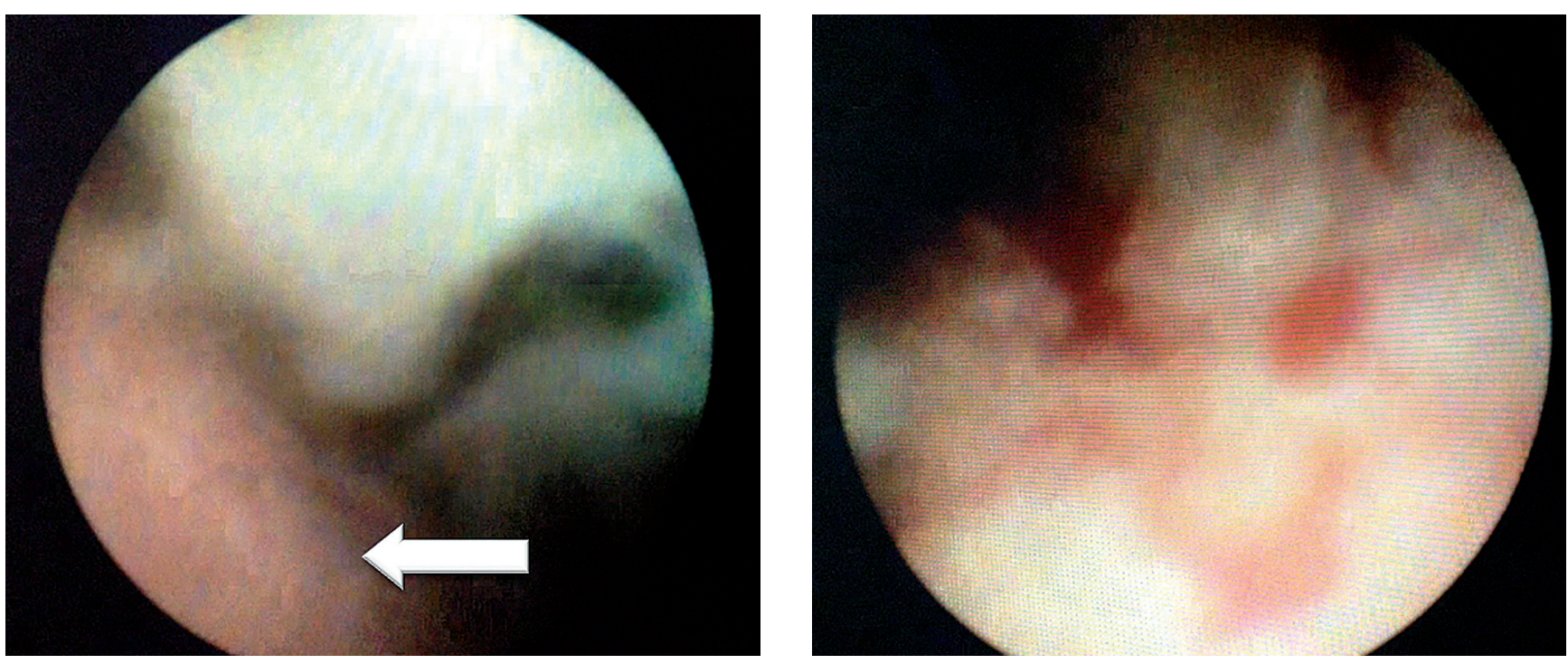

Figura 4. La imagen de la izquierda muestra la pared del colédoco liso, nacarado normal (flecha) y en la parte superior, el moco que llena la luz del colédoco. La imagen de la derecha muestra la pared del colédoco irregular, con puntos hemáticos, compatible con las papilas productoras de moco que caracterizan este tumor, de la cual se toma una biopsia con Spybite, dando positiva para NMPI.

asociado. Esta clasificación es similar a la de NMP del páncreas, ya que se asume que tienen una secuencia similar en su formación (10).

Las manifestaciones clínicas más comunes son el dolor abdominal intermitente, la colangitis aguda e ictericia (4, $11)$, pero con cierta frecuencia $(29,5 \%)$ los pacientes no tienen síntomas (12-14). Alrededor del 30\% de ellos tienen una historia previa o la existencia concomitante de cálculos biliares, como se muestra en los informes de los países del Lejano Oriente y como pasó en nuestra paciente (15), aunque no es así en los países occidentales (12). La localización del tumor varía: algunos informes muestran que la mayoría de NMPVB se encuentra en el conducto biliar intrahepático $(15,16)$, mientras que otro mostró que la ubicación más común es el hilio hepático (12).

En cuanto a las herramientas diagnósticas además de la CPRE, como se ve en este reporte, la colangioscopia peroral por el sistema Spyglass puede tener un rol fundamental dado que permite mirar la vía biliar directamente, evaluar la extensión del tumor a lo largo del epitelio biliar y tomar inmediatamente la biopsia con una pinza que puede sacar muestra de las papilas para proporcionar información precisa y elegir así el tratamiento adecuado (17).

En conclusión, no todo paciente que presente síndrome obstructivo biliar tiene cálculos, se debe sospechar de NMPVB, ya que es una lesión premaligna que, si se detecta y se trata a tiempo, es una enfermedad curable; al contrario del colangiocarcinoma, el cual puede evolucionar si no se trata a tiempo.

\section{REFERENCIAS}

1. Kubota K, Nakanuma Y, Kondo F, et al. Clinicopathological features and prognosis of mucin-producing bile duct tumor and mucinous cystic tumor of the liver: A multi-institutional study by the Japan Biliary Association. J Hepatobiliary Pancreat Sci. 2013;39:123-130.

2. Minagawa N, Sato N, Mori Y. A comparison between intraductal papillary neoplasms of the biliary tract (BTIPMNs) and intraductal papillary mucinous neoplasms of the pancreas (P-IPMNs) reveals distinct clinical manifestations and outcomes. EJSO. 2013;39:554-8.

3. Kim BS, Joo SH, Lim SJ, et al. Intrahepatic Biliary Intraductal Papillary Mucinous Neoplasm With Gallbladder Agenesis: Case Report. Surg Laparosc Endosc Percutan Tech. 2013;23:e61-4.

4. Barton JG, Barrett DA, Maricevich MA, et al. Intraductal papillary mucinous neoplasm of the biliary tract: A real disease? HPB. 2009; 11:684-91.

5. Chang WF, Huang GS, Chang WC. An unusual but important cause of biliary obstruction. Gastroenterology. 2013;145:59-62.

6. Lim JH, Jang KT, Choi D. Biliary intraductal papillary mucinous neoplasm manifesting only as dilatation of the hepatic lobar or segmental bile ducts: imaging features in six patients. AJR. 2008;191:778-82.

7. Lim JH, Yi CA, Lim HK, Lee WJ, Lee SJ, Kim SH. Radiological spectrum of intraductal papillary tumors of the bile duct. Korean J Radiol. 2002;3(1):57-63.

8. Zen Y, Fujii T, Itatsu K, et al. Biliary papillary tumors share pathological features with intraductal papillary mucinous 
neoplasm of the pancreas. Hepatology. 2006;44(5):133343.

9. Nakanuma Y, Curado M, Fransceschi S, et al. "Intrahepatic cholangiocarcinoma" in WHO Classification of tumours of the digestive system. Bosman F, Carneiro F, Hruban RH, Theise ND (editors). 4th edition. Lyon, France: IARC; 2010. p. 217-27.

10. Zen Y, Fujii T, Itatsu K, et al. Biliary cystic tumors with bile duct communication: A cystic variant of intraductal papillary neoplasm of the bile duct. Modern Pathol. 2006;19(9):1243-54.

11. Ohtsuka M, Kimura F, Shimizu H, et al. Similarities and differences between intraductal papillary tumors of the bile duct with and without macroscopically visible mucin secretion. Am J Surg Pathol. 2011;35(4):512-21.

12. Rocha FG, Lee H, Katabi N, et al. Intraductal papillary neoplasm of the bile duct: A biliary equivalent to intraductal papillary mucinous neoplasm of the pancreas? Hepatology. 2012;56(4):1352-60.
13. Onoe S, Shimoyama Y, Ebata T, et al. Prognostic delineation of papillary cholangiocarcinoma based on the invasive proportion: A single-institution study with 184 patients. Surgery. 2014;155(2):280-91.

14. Yeh C, Jan Y, Yeh T, Hwang T, Chen M. Hepatic resection of the intraductal papillary type of peripheral cholangiocarcinoma. Ann Surg Oncol. 2004;11(6):606-11.

15. Kim KM, Lee JK, Shin JU, et al. Clinicopathologic features of intraductal papillary neoplasm of the bile duct according to histologic subtype. Am J Gastroenterol. 2012;107(1):118-25.

16. Jung G, Park KM, Lee SS, et al. Long-term clinical outcome of the surgically resected intraductal papillary neoplasm of the bile duct. J Hepatology. 2012;57(4):787-93.

17. Yang J, Wang W, Yan L. The clinicopathological features of intraductal papillary neoplasms of the bile duct in a Chinese population. Digest Liver Dis. 2012;44(3):251-6. 